\title{
Модель формирования доверия онлайн-потребителей к сфере электронной торговли
}

\author{
Цао Цзыин, Елена Тюрина*, Александр Филатов \\ Дальневосточный федеральный университет, Владивосток, Россия
}

\author{
Информация о статье \\ Поступила в редакичю: \\ 25.03.2020 \\ Принята \\ к опубликованию: \\ 14.04.2020 \\ УДК 658.8.012.12; 658.89; \\ 65.012 .12 \\ JEL M31
}

\section{Ключевые слова:}

доверие онлайн-

потребителей, механизм

отзывов, механизм возврата

(обмена) товара, механизм

обеспечения онлайн-

гарантий, социальное при-

сутствие, глобальность опы-

та онлайн-покупок, культура

\section{Keywords:}

consumers e-confidence, mechanism of reviews, mechanism of commodities return (exchange), mechanism of providing online guarantees, social presence, global online purchase experience, culture

\begin{abstract}
Аннотация
Сделав анализ результатов многих теоретических и эмпирических исследований (1999-2018 г2.), мы обнаружили, что в области доверия онлайн-потребителей все ещуе существуют противоречия и разрывы. Наше текущее исследование расширяет модель D. Gefen и D.W. Straub тремя механизмами формирования доверия (механизмы отзывов, возврата и обмена товара, обеспечения онлайн-гарантий), которые рассматриваются как предшественники доверия. Встраивая в модель доверия глобальность опьта онлайнпокупателей как фактор потребительского поведения, а также культуру как контрольную переменную, мы предлагаем более точную оценку факторов, которая поможет внести ясность в понимание того, как формируется доверие онлайн-покупателей в современном контексте трансграничной электронной торговли. Мы предполагаем, что восприятие сочиального присутствия продолжает влиять на формирование доверие онлайн-потребителей с опытом зарубежных онлайн-покупок.
\end{abstract}

Model for Creating Online Consumers Confidence in E-commerce

Cao ZiYing, Elena Tyurina, Alexander Filatov

\section{Abstract}

Having analyzed the results of many theoretical and empirical studies (1999-2018), we found that contradictions and gaps in the field of online consumers confidence still existed. Our current research extends the model of D. Gefen and D.W. Straub with three mechanisms of creating confidence (mechanism of review, return and exchange of commodities, providing online guarantees), which are regarded as predecessors of confidence. By fitting the online consumers global experience as a factor of the consumers behavior, as well as the culture as a control variable into the confidence model, we suggest a more accurate assessment of factors that will help to clarify the understanding of how the consumers e-confidence is created in the modern context of cross-border e-commerce. We assume that understanding of 2 he social presence is still influencing on creating

* Автор для связи: tyurina.ea@dvfu.ru

DOI: https://dx.doi.org/10.24866/2311-2271/2019-4/42-59 
the confidence of the online consumers having the experience in foreign online purchasing.

\section{Введение}

Сторона предложения в трансграничной электронной торговле характеризуется высокими темпами развития, что открывает ритейлерам разнообразные возможности для роста бизнеса на международном рынке. Сторона спроса - тем, что онлайн-потребители сталкиваются с новой средой онлайнтранзакций, огромными массивами информации, неструктурированными отзывами, более сложным и слабо контролируемым процессом возврата (обмена) товаров, более высокими рисками, сложным управлением личной информацией и процессом онлайн-платежей, что отражается на формировании и поддерживании доверия онлайн-потребителей. В этом случае изучение поведения онлайн-потребителей может помочь нам лучше понять механизмы формирования доверия.

Тщательное рассмотрение существующих моделей доверия онлайнпотребителей, различных теоретических положений, на которых они основаны, стало для нас важной задачей для построения более точной модели, учитывающей контекст трансграничной электронной торговли.

Доверие - решающий фактор развития электронной торговли: ощущая доверие, онлайн-потребители преодолевают воспринимаемые риски, легко находят и обрабатывают информацию о товарах и услугах, предоставляемых онлайн-продавцом, что подталкивает их к совершению онлайн-покупки. Проблематика доверия онлайн-потребителей активно изучается, благодаря исследованиям D.H. McKnight и соавторов $(2001,2002)$ [1, 2], D. Gefen $(2000,2002)[3,4]$, D. Gefen и D.W. Straub (2004) [5], P.A. Pavlou и D. Gefen (2004) [6], Thompson S.Н. Тео и соавторов (2007) [7], P. Palvia (2009) [7], Jung Kun Park и соавторов (2012) [8], I.B. Hong и H.S. Cha (2013) [9], S. Kim и Н. Park (2013) [10], Е. Toufaily и соавторов (2013) [11] и др., мы понимаем, как происходит формирование доверия онлайн-потребителей, их восприятия относительно факторов, предшествующих доверию («факторы-предшественники»), а также как доверие влияет на намерения онлайн-потребителей. Одновременно стремительная глобализация электронной торговли непрерывно расширяет пространство выбора онлайн-шопинга, происходит переплетение различных культурных контекстов, что снова создает барьеры на пути формирования доверия потребителей. Благодаря развитию онлайн-транзакций между странами потребительский опыт онлайн-покупок становится более богатым.

Именно в этом контексте изучение предшественников доверия онлайнпотребителей различных стран с учетом дифференцирования опыта онлайнпокупок поможет понять, насколько существующие модели объясняют восприятие факторов доверия, какие противоречия ограничивают понимание поведения потребителя в условиях развития трансграничной электронной торговли.

В литературе известно более 15 моделей доверия онлайн-потребителей. Одни из самых известных - модель D.H. McKnight и соавторов $(2001,2002)[1$, 2], основанная на восприятии репутация онлайн-продавца, восприятии качества интернет-магазина и гарантии безопасности онлайн-транзакций; модель D. Gefen и D. W. Straub $(2000,2002,2004)$ [3-5], основанная на взаимосвязи доверия с уровнем знакомства с интернет-магазином, уровнем склонности к доверию и восприятием социального присутствия; модель S.L. Jarvenpaa (1999) [12] 
и модель Thompson S.H. Тео (2007) [13], построенные с учетом восприятия репутации онлайн-продавца, размера интернет-магазина, гарантии безопасности онлайн-транзакций и культурного контекста. Однако результаты эмпирических исследований открывают для нас ряд противоречий и разрывов (Danny Tengti Kao, 2009; Pitney Bowes, 2016; Brightlocal, 2016; 100ec, 2017; Worldpay, 2017; Mypinpad, 2017; Askci, 2017; Forrester, 2018) [14-21].

Сравнительные исследования взаимосвязи культурных отличий (по параметрам модели Hofstede, как контрольная переменная в модели) и доверия онлайн-потребителей носят противоречивый характер. Chai L. и Pavlou P. (2002) [22], Jung Kun Park и соавторы (2012) [8] установили взаимосвязь между разными культурами и доверием онлайн-потребителей, проведя исследования на примере Китая и США, США и Южной Кореи. Однако S.L. Jarvenpaа и Tractinsky N. (1999) [12], Danny Tengti Kao (2009) [14] не установили сильных культурных отличий в антецедентах доверия, исследования проведены на примере Австралии, Израиля и Финляндии, и, соответственно, Австралии и Тайваня.

Изучив методологии вышеуказанных исследований и их результаты, мы посчитали данное противоречие методической ошибкой встраивания фактора культуры в модель доверия. Большинство исследователей доверия онлайнпотребителей в контексте различных культур использовали параметры модели Hofstede [23] для измерения культуры. При этом Hofstede изначально разработал свою модель влияния культурных ценностей на ценности на рабочем месте, что создает ограничения на распространение метрик и шкал модели на сравнительные исследование доверия онлайн-потребителей в различных культурных контекстах. Отметим, что наши предыдущие исследования позволяют предположить, что доверие онлайн-потребителей в России и Китае имеет значительные отличия [15].

Помимо этого, в условиях развития трансграничной электронной торговли фактор восприятия социального присутствия (модель D. Gefen), иначе его можно назвать фактором социальной принадлежности онлайн-потребителя к электронной сети (среде онлайн-торговцев), на наш взгляд, требует дополнительного изучения. Кроме того, одними из недооцененных факторовпредшественников доверия являются механизмы формирования доверия, что связано с бурным развитием современных технологий наряду с изменением степени глобальности опыта онлайн-потребителей.

Технологически электронная торговля, значительно изменившись за последние 10 лет, не просто открывает для покупателя новые возможности, она вовлекает его в свои процессы, что в свою очередь подталкивает нас расширить границы механизмов формирования доверия [24]. Например, механизм обеспечения онлайн-гарантий, работа которого исследована D.H. McKnight и соавторами (2002) [1], Thompson S.H. Тео и соавторами (2007) [13], S. Kim и H. Park (2013) [10], представлен в роли надежного сигнала, обеспечивающего безопасность. Однако с точки зрения современной практики электронной торговли, данный механизм организует сетевой сервис, постоянно предоставляя информацию о предупреждении безопасности, направляя онлайн-потребителей избегать риска и использовать технологии защиты личной информации, своего аккаунта в процессе онлайн-покупок.

Постоянно обогащающийся опыт онлайн-покупок, в том числе на зарубежных платформах и интернет-магазинах, подталкивает нас к уточнению по- 
нимания работы механизма отзывов, рассматривая его не только как высоконадежный сигнал, но и источник качественной информации о товарах и услугах онлайн-продавца.

Возврат (обмен) товара постепенно превратился в послепродажный сервис, предоставляемый онлайн-продавцом в качестве компенсации за утраченное доверие к онлайн-продавцу, а не просто как обещание онлайн-продавца, как это учитывается в работах Bonifield и др, (2010) [25]; Z. Pei и др., (2014) [26]. Кроме того, механизм возврата (обмена) товара работает с использованием современных технологий, позволяя онлайн-потребителям осуществлять контроль на любом шаге процесса возврата (обмена) товаров.

Когда онлайн-потребители хорошо знакомы со сферой онлайнтранзакции, полностью понимают заботу и помощь онлайн-продавца, механизмы доверия способствуют принятию решения о покупке. Однако, когда онлайн-потребитель хочет купить товар за рубежом, изменение онлайн-среды создает новые сложности для иностранных онлайн-потребителей, возникают такие барьеры, как языковая коммуникация, новые способы онлайн-платежа или отличающиеся правила, расстояние и доставка товаров и т.д., которая заставляет их снова почувствовать неопределенность. В этой ситуации, мы предполагаем, что восприятие социального присутствия возможно продолжает влиять на формирование доверие онлайн-потребителей с опытом зарубежных онлайн-покупок. А также, оно может оказывать большее влияние по сравнению со степенью влияния на доверие онлайн-потребителей без опыта зарубежных онлайн-покупок.

Развитие электронной торговли в глобальном масштабе постоянно увеличивает богатство потребительского опыта зарубежных онлайн-покупок. По данным Pitney Bowes, более 66\% онлайн-потребителей в всем мире имеют зарубежный опыт онлайн-покупок [15]. В начале распространения трансграничной онлайн-торговли потребители часто делали зарубежные онлайн-заказы по причине разнообразного ассортимента, качественных товаров и низких цен (AКИТ; iResearch, 2012-2014) [27-29]. В дальнейшем безопасность платежей, репутация электронной платформы, отслеживание онлайн-заказов и качество обслуживания клиентов стали главными факторами, формирующими доверие и намерение совершать онлайн-покупки на зарубежных платформах и магазинах (iResearch, 2015-2016) [30]. Эта ситуация побуждает нас задуматься: помимо влияния продолжительности опыта онлайн-покупок или количества онлайнпокупок на доверие потребителей (Liu, Dixon и Murphy, 2002; Gounaris и Venetis, 2002) [31, 32], как опыт онлайн-покупок определяется с глобальной точки зрения, как будет формироваться доверие по мере повышения степени глобальности потребительского опыта.

Таким образом, модели формирования доверия онлайн-потребителей, которые использовались в других исследованиях, пока не совершенны. Между результатами многих теоретических и эмпирических исследований факторов доверия все еще существуют противоречия и разрывы. Наша цель - расширить модель D. Gefen и D.W. Straub, чтобы повысить точность оценки факторовпредшественников доверия, которая сможет применяться в различных культурных контекстах с учетом глобальности опыта онлайн-покупок.

Мы используем в исследовании модель D. Gefen и D.W. Straub в связи с тем, что: a) 4 детерминанты (восприятие честности, доброжелательности, способности и предсказуемости) для измерения самого доверия, предложенные 
данными учеными, уже верифицированы во многих эмпирических исследованиях; б) результаты исследований D. Gefen и D.W. Straub широко цитируются исследователями в качестве теоретической основы для изучения доверия онлайн-потребителей; в) эффект социального присутствия требует переосмысления, его повторное исследование открывает новые возможности уточнения модели доверия онлайн-потребителей в условиях применения современных технологий; г) D. Gefen и соавторы рекомендуют проведение дальнейших исследований доверия онлайн-потребителей, в том числе с учетом влияния воспринимаемого риска и безопасности, качества товаров и обслуживания.

\section{Теоретическая рамка исследования}

Чтобы улучить понимание того, как формируется доверие онлайнпотребителей в различных культурных контекстах с учетом изменения степени глобальности опыта онлайн-покупок, мы будем развивать обсуждение вокруг важных факторов-предшественников формирования доверия, в том числе механизмов отзывов, возврата (обмена) товаров, обеспечения онлайн-гарантии, а также восприятия социального присутствия с учетом детерминантов самого доверия. В то же время мы пересмотрим подход к встраиванию и измерению культуры в модели доверия онлайн-потребителей.

Что понимают под доверием онлайн-потребителей и как его измерить? Доверие онлайн-потребителей, как многомерная конструкция, состоит, согласно D. Gefen и D.W. Straub (2004), из четырех детерминант: честность, доброжелательность, способность и предсказуемость [5], и определяется восприятием поведения онлайн-продавца. Восприятие честности указывает на то, что в понимании покупателя онлайн-продавец может выполнить свои обещания; восприятие доброжелательности означает, что онлайн-продавец часто выражает активную заботу о потребностях потребителя; восприятие способностей продавца показывает, что онлайн-продавец обладает достаточной силой обеспечить качественное обслуживание; восприятие предсказуемости действий продавца указывает на то, что потребитель легко спрогнозирует действия онлайнпродавца.

Социальное присутствие, как фактор модели доверия онлайнпотребителей, используется нами в качестве абстрактного психологического термина (social presence), под которым понимается социальная принадлежность потребителя к электронной сети (среде онлайн-торговца). Когда онлайнпотребитель не ощущает своей принадлежности к электронной сети, ему трудно почувствовать реальные межличностные отношения в сфере электронной торговли. В этом случае он ощущает неопределенность, доверие к онлайнпродавцу трудно формируется (D. Gefen и D.W. Straub, 2004). Однако когда онлайн-потребители воспринимают социальное присутствие (они часть электронной сети) благодаря: (1) применению видео, графических и текстовых объяснений, фотографий, видео-презентаций товаров, которые помогают им найти нужную информацию; (2) работе онлайн-чата и электронной почты для быстрой передачи запросов и ответов; (3) предоставлению широких возможностей в процессе онлайн-транзакций, в том числе для выражения мнений (отзывов) и др., они определенно ощущают себя участниками сети, вовлеченными в деятельность и развитие электронной платформы и / или магазина, и легко доверяют онлайн-продавцу.

Согласно модели D. Gefen и D.W. Straub (2004), восприятие социального 
присутствия онлайн-потребителя действительно помогает повышать уровень доверия. Честность и предсказуемость поведения онлайн-продавцов оказались необходимыми предпосылками для потребительских намерений онлайнпокупки. При этом доброжелательность и способность онлайн-продавца не показала сильного влияния на намерения покупки онлайн-потребителей. Уровень склонности к доверию, как контрольная переменная в модели D. Gefen и D.W. Straub (2004), оказывает большое влияние на доверие.

С учетом обозначенных выше противоречий и разрывов, мы предлагаем расширить модель D. Gefen и D.W. Straub, добавив к фактору социального присутствия такие факторы-предшественники, как (1) механизм отзывов товара; (2) механизм возврата (обмена) товара; (3) механизм обеспечения онлайнгарантии, а также фактор культуры как контрольную переменную, и фактор потребительского поведения - глобальность опыта онлайн-покупок.

Механизм формирования доверия рассматривается нами как система, которая работает благодаря эффективному взаимодействию нескольких элементов, побуждает потребителя к нужным действиям и, соответственно, формирует и поддерживает доверие (Цао Цзыин, 2018) [33].

Механизм отзывов товара (их количество и контекст, поставленные оценки, повторные отзывы, размещаемые фотографии и т.д.), который в настоящее время является одним из стандартов электронной розничной торговли, рассматривается нами как высоконадёжный, правильно воспринимаемый и интерпретируемый потребителями сигнал (Metzger, 2006; Donath, 2007) [34, 35]. Теория сигналов в экономике утверждает, что, когда между покупателями и продавцами возникает серьезная асимметрия в информации, люди часто полагаются на сигналы, если им приходится судить о ненаблюдаемом качестве (Spence, 1973) [36]. Онлайн-потребители воспринимают свойства информации о товарах и услугах как высококачественный сигнал, заложенной предыдущими покупателями в систему отзывов, которые рассматриваются как очень важные основания при принятии решений в процессе онлайн-транзакций.

Механизм возврата (обмена) товара, также, как и другие механизмы, является многоэлементным, включает сервисы электронной платформы или магазина, предоставляемые онлайн-потребителям в рамках возврата и обмена товара. Как правило, исследователи, изучающие различные аспекты сервисной политики, опираются на теорию качества сервиса (A. Parasuraman, и др., 1988; J.J. Cronin и S.A. Taylor, 1992; S.R. Muhammad и др., 2012) [37-39], измеряя степень разницы между действительно воспринимаемым и ожидаемым потребителем уровнем обслуживания.

Однако отмеченными нами несоответствия между обещаниями возврата (обмена) товара онлайн-продавца и фактическим процессом возврата (обмена) товара (например, онлайн-продавец не признает проблемы качества своего товара, и отказываются принять сервис возвращения (обмена); онлайн-продавец намеренно задерживает возврат (обмен) товаров и т.д.) (100ес, 2017) [17], наше исследование сделает акцент на сервисе после продажи. При оценке механизма возврата (обмена) товара, теория качества сервиса уже не может объяснить данные проблемы.

В условиях усложнения сферы электронной торговли онлайнпотребители обращают внимание на сам процесс и результат возврата (обмена) товара, а также взаимодействие с онлайн-продавцом. Восприятие онлайнпотребителем данных факторов можно объяснить с помощью теории справед- 
ливости. Теория справедливости, впервые предложенная Adams (1965) [40] и получившая развитие благодаря работам R.J. Bies и J.S. Moag (1986) [41], указывает на то, что чувство справедливости возникает относительно двух взаимосвязанных областей: 1) дистрибуция, где формируется дистрибутивная справедливость, которая фокусируется на правилах и социальных нормах, касающихся распределения; 2) процедуры, где формируется процедурная справедливость, фокусирующаяся на процессе принятия решений и процедурах, используемых для достижения результатов решения; 3) коммуникации, где формируется интерактивная справедливость, фокусирующаяся на межличностных отношениях, включая предоставление информации и адекватность объяснений. Как раз в соответствии с теорией справедливости, воспринимаемая интерактивная справедливость, а также процедурная и дистрибутивная справедливости в процессе возврата (обмена) товара помогут восстановить потерю доверия онлайн-потребителей и сделать залог их повторных покупок в будущем.

Согласно теории запланированного поведения I. Ajzen [42], люди с большей вероятностью предпримут ожидаемые действия в случае, если степень воспринимаемого контроля над таким действием будет высока. Рассматривая механизм возврата (обмена) товара через теоретическую рамку запланированного поведения, мы полагаем, что если данный механизм работает таким образом, что онлайн-потребителю легко отслеживать возврат (обмен), то поведение онлайн-продавца в процессе возврата (обмена) будет восприниматься как легко прогнозируемое и находящееся под контролем.

Механизм обеспечения онлайн-гарантий рассматривается нами как надежный сигнал для снижения неопределенности онлайн-потребителей, который помогает им сформировать доверие и стимулирует их делать онлайнпокупки (D. Miyazaki, A. Fernandez, 2001; K.M. Kimery и M. McCord, 2002; A. Noteberg и др., 2003; D.J Kim и др., 2004) [43-46]. Поскольку потребители все равно сталкиваются с опасными ситуациями (мошенничество, фишинговые ссылки и т.д.) во время онлайн-покупки (Worldpay, 2017; 199it, 2017) [18, 47], наше исследование переключает внимание на механизм обеспечения онлайнгарантий не только как предпродажные обещания, но и как качественный сетевой сервис. В соответствии с теорией качества сетевого сервиса (А. Parasuraman и др., 2005; R. Ladhari, 2010; Р. Thaichon и др., 2014) [48-50], воспринимаемое клиентами качество сервиса определяется комплексной оценкой качества веб-сайта, качества информации, клиентского обслуживание, гарантии безопасности/конфиденциальности и др. Особенно гарантия безопасности и конфиденциальности показывают значительное влияние в контексте особенностей сетевой сферы. Высококачественный сетевой сервис, воспринимаемый онлайн-потребителями в процессе обслуживания как безопасный и конфиденциальный, может рассматриваться как надежный сигнал, напоминающий онлайн-потребителям о том, что онлайн-продавец поможет максимально снизить вероятность возникновения риска. Сетевой сервис такого уровня устраняет беспокойства онлайн-потребителей и способствует формированию доверия.

С учетом рассмотренного методического противоречия, при встраивании культурного фактора в модели доверия мы предложили использовать для измерения культуры более подходящую по контексту и своим параметрам для исследования доверия теорию социальных аксиом, которая подробно рассмотрена в работе Н.М. Лебедева и А.Н. Татарко (2009) [51]. Мы воспользовались их работой, чтобы перепроверить, как доверие онлайн-потребителей формируется 
в разных культурных контекстах.

С точки зрения Leung и соавторов (2002) [52], «социальные аксиомы» понимаются как «генерализованные верования о себе, социальной и физической среде, духовном мире, высказанные в форме утверждений об отношениях между реально существующими фактами или идеями». Это означает, что поведение людей может определяться верованием, которое указывает на то, что люди дают свое собственное восприятие взаимосвязи вещей (две вещи могут быть любыми сущностями, а связи могут носить причинный или корреляционный характер ) в окружающей среде.

Доверие же в свою очередь рассматривается как верование потребителей в то, что компании будут действовать, исходя из лучших интересов потребителей, и придерживаться своих обещаний (J. Kang, 2014) [53]. Вероятно, российские и китайские онлайн-потребители, находящиеся под влиянием их различных культурных контекстов в течение длительного времени, сформировали свои собственные уникальные утверждения о связи одного реальной вещи с другой. Следовательно, теория социальных аксиом, которая максимально ориентирована на веровании человека, применяется в нашей модели формования доверия онлайн-потребителей для измерения фактора культуры.

Социальные аксиомы включают в себя 5 измерений. Первые три - «социальный цинизм», отражающий негативный либо позитивный взгляд на природу человека; «социальная сложность», указывающая на уверенность во множестве или единственности пути достижения цели; «религиозность», указывающая на существование веры в существование сверхъестественного или ее отсутствие, могут непосредственно отразить отношение людей, находящихся под влиянием внешней среды в течение длительного времени. Также они помогут изучить формирование доверия онлайн-потребителей в различных культурных контекстах.

Остальные два измерения - «награда за усилия» указывает на убежденность в предопределенности жизненных событий либо в том, что человек сам определяет свой жизненный путь; «контроль судьбы» отражает уверенность (или ее отсутствие) в том, что усердие «по жизни» вознаграждается, представляют собой уверенность людей в получении вознаграждения после собственных усилий [52].

В сфере электронной торговли потребители могут участвовать в деятельности и развитии электронной платформы, магазина, оставляя отзывы о своих покупках и предоставленном сервисе, выражая чувства собственного опыта онлайн-покупки. Это побуждает онлайн-продавцов постоянно улучшать свои товары и услуги, чтобы добиться доверия онлайн-потребителей. Измерения «награда за усилия» и «контроль судьбы» тоже подходят к нашему исследованию.

По результату исследования Bуoungho Jin и соавторы (2006) [54], уровень опыта онлайн-покупок определяет доверие потребителей: более опытные покупатели гораздо легче доверяют электронной торговле благодаря тому, что знакомая сфера онлайн-шопинга заставляет их не сомневаться в гарантии безопасности и конфиденциальности, выполнении заказов и рекламных акциях. Потребительский глобальный опыт отражает ценностную тенденцию группы потребителей со схожими потребностями, вкусами и образом жизни, которые выходят за пределы национальных и культурных границ (HaeJung Kim и соавторы, 2014) [55]. Что касается шопинга на зарубежных электронных платфор- 
мах и магазинах, потребители, не имеющие такого опыта, предпочитают делать онлайн-заказы через посредников (Nielsen, 2016) [56]. По мере того, что онлайн-потребители начинают сами совершить онлайн-заказ на зарубежных электронных платформах (Data Insight, 2018) [57], они уже имеют опыт онлайнпокупок высокой степени глобальности.

Эксперты iResearch, Nielsen и Data Insight рассматривают три канала онлайн-покупок, соответственно, три типа опыта:

1) опыт онлайн-покупок только в своей стране;

2) опыт онлайн-покупок, сделанных на зарубежных электронных платформах / магазинах через посредников;

3) опыт онлайн-покупок, сделанных на зарубежных электронных платформах / магазинах самостоятельно.

Мы полагаем, что по мере повышения степени глобальности опыта онлайн-покупок, предпосылки, по которым онлайн-потребители доверяют онлайн-продавцам также меняются.

\section{Факторы модели формирования доверия онлайн-потребителей}

Предлагаемая модель доверия (рис. 1), применительно к различным культурным контекстам и с учетом уровня глобальности опыта онлайнпотребителей, объясняет, как механизмы формирования доверия, в работе которых непосредственно участвуют покупатели, приводят к эффекту социального присутствия потребителей в сети, что в свою очередь, а также, возможно, напрямую, создает онлайн-доверие.

В нашей работе, опираясь на комплексную теоретическую рамку, включающую положения теории сигналов, теории качества сервиса, теории справедливости, теория запланированного поведения и теория качества сетевого сервиса, а также исследование практики электронной розничной торговли, мы выдвинули ряда гипотез относительно того, как онлайн-потребитель реагирует на качество информации (на странице товара), что происходит с его доверием в случае большого объема отзывов и сложной структуры их размещения (на электронных платформах / магазинах); как онлайн-потребитель ощущает справедливость и контролируемость процесса возврата (обмена) товара после продажи, что происходит с его доверием в случае несоответствия обозначенных онлайн-продавцом / платформой правил возврата (обмена) товара и фактической ситуации на различных этапах процесса возврата (обмена) товара; как онлайн-потребитель реагирует на качество сетевого сервиса, предоставляемого за счет работы механизма обеспечения онлайн-гарантии, что происходит с его доверием в случае раскрытия личной информации, ситуаций мошенничества и введения в заблуждение.

Онлайн-потребитель, столкнувшись с отзывами на веб-странице товара, реагирует на качество информации (содержащейся в них), которое, согласно теории сигналов, воспринимается как высоконадёжное основание покупки. На этом этапе (поиск и принятие решения о покупке) эффект доверия либо возникает и растет, либо снижается. Данный эффект усиливается в том случае, если количество отзывов на веб-странице товара велико (превышает 1 страницу). Эффект будет увеличиваться, если механизм отзывов предоставляет релевантную информацию о товарах и услугах, сопровождает текстовую информацию сигнализирующими символами, фотографиями, дополнительными комментариями и т.д. 
Так происходит потому, что онлайн-потребители сначала выбирают и читают несколько отзывов. Они, как правило, не хотят читать более 10 отзывов (Brightlocal, 2016) [16]. Онлайн-потребитель с трудом доберется до интересных для него отзывов, если информация, например, неструктурированная.

Если механизм отзывов предоставляет потребителю свежую информацию о товарах и услугах, мы предполагаем, что эффект доверия будет увеличиваться. Так как по данным Brightlocal [16], 73\% потребители считают, что отзывы старше 3 месяцев больше не актуальны. Если потребитель видит отзывы, возраст которых превышает год, они будут иметь меньшее влияние на решение о покупке.

Если механизм отзывов предоставляет правильное и точное описание товаров и услуг, эффект доверия онлайн-потребителей будет увеличиваться. Помимо письменных, отзывы с прикрепленными фотографиями и видео реальных товаров (например, на электронной платформе Tаобао, Amazon, eBay) позволяют потребителю более точно понять характеристики товара, предоставляют онлайн-потребителям возможность рассматривать товары в реальной ситуации (обстановке).

Эффект доверия будет увеличиваться, если механизм отзывов предоставляет комплексную информацию о товаре и услугах. Так происходит потому, что онлайн-потребители, особенно с большим опытом онлайн-покупок, часто стремятся максимально подробно узнать характеристики товара, например, восприятие материала на ощупь, запах, размер обуви стандартный или нет и т.д., которые трудно осознать через фотографии / видео реальных товаров.

Возможность быстро найти такую информацию среди отзывов помогает увеличить эффект доверия.

Таким образам, мы предполагаем, что если механизм отзывов «выдает» высококачественную информацию (релевантную, свежую, точную и комплексную), то онлайн-потребители ощущают существование реального межличностного общения во время поиска товара, и у них быстро развивается чувство принадлежности к сайту, которое, в свою очередь, активно повышает эффект доверия.

Основываясь на теории качества сервиса, мы предполагаем, что онлайнпотребитель воспринимает возможность возврата и обмена товара как обязательный элемент обслуживания, при котором он (потребитель) получает высококачественный сервис и возможность вести контроль на всех этапах возврата и ощущает, согласно теории Адамса и Айзен (Ajzen), справедливое отношение к себе и воспринимаемый контроль над действиями продавца и платформы.

Как правило, онлайн-потребители, поняв необходимость возврата (обмена) хотят получить консультацию онлайн-продавца. В случае, если онлайнпродавец быстро вышел на связь в чате, вежливо отреагировал на вопросы покупателя, выразил заботу о его интересах, проявил терпение в объяснении оформления возврата (обмена) товара, у онлайн-потребителя будет возникать хорошее впечатление о поведении продавца, а взаимодействие и отношения будут оцениваться как качественные и доброжелательные. 


\begin{tabular}{|c|}
\hline Механизм отзывов \\
Bоспринимаемое качество ин- \\
формации \\
Релевантная \\
Свежая \\
Точная \\
Комплексная
\end{tabular}

Механизм возврата (обмена) товаров

1) Воспринимаемая справедливость

- Интерактивная справедливость

- Процедурная справедливость

- Дистрибутивная справедливость

2) Воспринимаемый контроль

ᄂ Комплексная

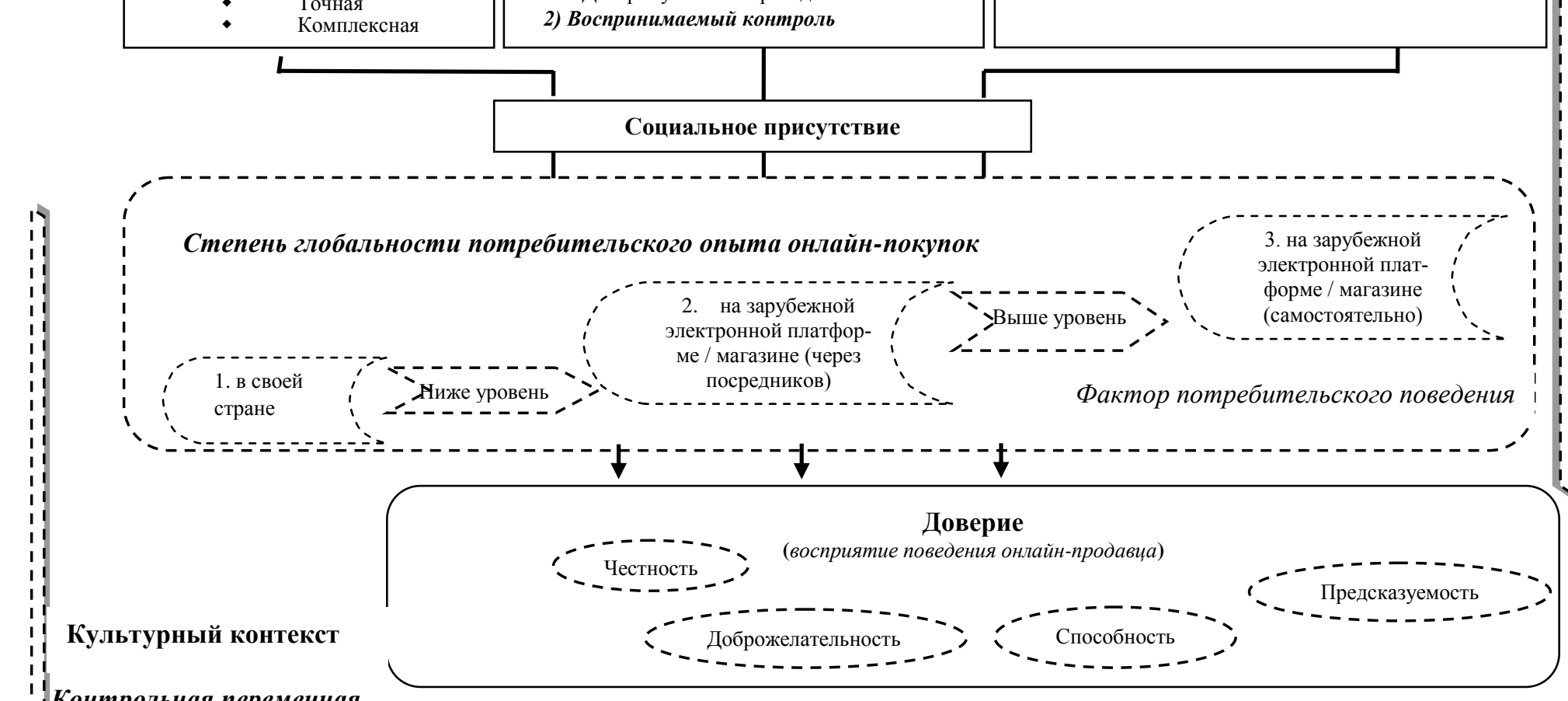

\section{Контрольная переменная}

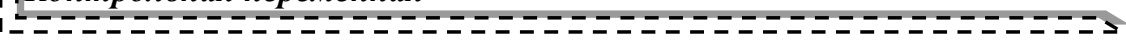

Puc. 1. Модель формирования доверия онлайн-потребителей

Источник: составлено авторами 
При входе в процессе подготовки заявки на возврат (обмен) товара, если онлайн-потребители могут быстро, успешно выполнить всю процедуру в несколько кликов без бумажных документов с поддержкой современных технологий на всех этапах (заполнение заявления (прилепленные доказательства), автоматический прием и рассмотрение заявки в короткий срок (например, 3 дня), отслеживание возврата и др.), онлайн-потребители будут ощущать данный процесс современным и простым. И они будут считать, что платформа и онлайн-продавец предоставляют им это удобство.

После того, как заявка на возврат (обмен) товара принята, если онлайнпотребители смогут получить соответствующие сервисы и условленные компенсации, как было обещано платформой и онлайн-продавцом, они будут воспринимать поведение онлайн-продавца как честное и смогут доверять.

После успешной подачи заявки на возврат (обмен), если онлайнпотребители смогут постоянно получать сигналы напоминания о том, как идет процедура возврата с помощью современных технологий, в течение всего времени до завершения заявки, принятия мер для решения проблемы и т.д., они будут воспринимать возврат как беспрепятственную процедуру.

Таким образом, мы предполагаем, что механизм возврата (обмена) товара происходит через предоставление вышеперечисленных сервисов, которые делают все процессы справедливыми (соответствующие интерактивной справедливости, процедурной справедливости и дистрибутивной справедливости) и контролируемыми. Это способствует тому, что онлайн-потребители воспринимают общение с онлайн-продавцам во время возврата как реальное и легко ощущают свое социальное присутствие в сети. В этом случай, эффект доверия будет увеличиваться, а утраченное доверие - восстанавливаться.

Согласно теории качества сетевого сервиса, Parasuraman и др., нам кажется, что онлайн-потребители, при получении обеспечения онлайн-гарантии для защиты их конфиденциальности и безопасности онлайн-сделки до и после онлайн-транзакций, часто воспринимают механизм обеспечения онлайнгарантии как высококачественный сетевой сервис.

До совершения онлайн-покупки, онлайн-потребители, как правило, наблюдают за наличием высоконадежных сигналов обеспечения онлайн-гарантии на электронной платформе, чтобы оценить возможность риска при проведении онлайн-сделки. Если онлайн-потребители увидели надежные сигналы (политика конфиденциальности и безопасности) на сайте, они будут считать, что их личная информация и аккаунт в целом находятся под защитой, а в случае онлайн-платежей, их онлайн-транзакции и личная информация о потребителях также защищены.

Однако новые опасные факторы в интернете возникают непрерывно. Всегда есть вероятность, что при проведении онлайн-сделки и после её окончания, когда онлайн-потребители не могут идентифицировать объявление или ссылки на мошенничество и т.д., они столкутся с раскрытием личной информации и мошенниками. Хотя эти причины не связаны с онлайн-продавцами, онлайн-потребители будут обвинять их в том, что они несвоевременно напомнили об опасной ситуации, и будут считать, что онлайн-гарантия не была обеспечена в полной мере. В результате их доверие к онлайн-продавцу будет потеряно.

В этой ситуации, если онлайн-продавец предоставляет не только надежные сигналы об обеспечении безопасности (политика конфиденциальности и 
безопасности), но и информацию о предупреждении безопасности до и после онлайн-сделки, механизм обеспечения онлайн-гарантия будет работать как качественный сетевой сервис. Например, активно рекомендовать онлайнпотребителям использовать более сложный пароль, никому не раскрывать его при проведении онлайн-покупок, напоминать обновлять пароль и систему безопасности, своевременно сообщать не доверять незнакомым ссылкам, каналам оплаты и информации о выигрыше. При такой поддержке онлайнпотребители будут быстро идентифицировать опасность, смогут ее избежать и будут воспринимать работу механизма обеспечения онлайн-гарантии как действительно надежно защищающую их личные интересы.

Таким образом, мы полагаем, что если механизм обеспечения онлайнгарантии происходит как предоставление качественного сетевого сервиса, то онлайн-потребители будут ощущать, что онлайн-продавец действительно принимает меры и взаимодействует с ними, чтобы обеспечить безопасность и конфиденциальность сферы онлайн-транзакций. В этом случае, онлайнпотребители будут легко воспринимать свою принадлежность к сайту, что увеличит эффект доверия.

В модели D. Gefen и D.W. Straub показано, что социальное присутствие в сети положительно влияет на эффект доверия онлайн-потребителей. Однако в нынешней ситуации все больше и больше онлайн-потребителей из разных стран совершают трансграничные онлайн-покупки, что создает ряд изменений и барьеров в сфере электронной торговли: языковая коммуникация, расстояние и срок доставки товаров (включая возврат/обмен), способ онлайн-платежа и т.д. Поддерживая идею D. Gefen, наша модель включает социальное присутствие, которое формируется с помощью механизмов, способствующих реальному комфортному человеческому взаимодействию. Мы будем перепроверять влияние социального присутствия на эффект доверия в разных культурных контекстах с учетом уровня глобальности опыта онлайн-потребителей. Таким образом, мы полагаем, что восприятие социального присутствия онлайнпотребители с разным опытом может складываться по-разному, соответственно, взаимосвязь механизмов формирования доверия с социальным присутствием и эффектом доверия также может отличаться.

Встраивание в нашу модель фактора культуры как контрольной переменной позволяет компаниям или онлайн-торговцам, которые занимаются или будут заниматься трансграничной электронной торговлей в разных странах, лучше овладеть культурными особенностями самих онлайн-потребителей и разобраться в кросс-культурных отличиях взаимосвязи механизмов формирования доверия, восприятия социального присутствия и эффекта доверия. Мы предполагаем, что наша модель предоставит практикам основания для более точной оценки эффективности инвестиций в создание и / или развитие элементов трансграничной электронной торговли, в том числе в технологии.

Предлагаемая нами модель отражает ряд гипотез (табл. 1), которые будут проверены на следующем, эмпирическом, этапе нашего исследования путем опроса 900 российских и 900 китайских онлайн-покупателей и оценки различных эффектов. 
Таблица 1

Гипотезы

\begin{tabular}{|c|c|}
\hline & \\
\hline $\begin{array}{c}\text { Номер } \\
\text { гипотезы }\end{array}$ & Гипотеза \\
\hline H1 & $\begin{array}{l}\text { Механизм отзывов товара, который предоставляет онлайн-потребителям } \\
\text { качественную информацию (релевантную, точную, свежую и комплексную), } \\
\text { показывает положительное отношение с эффектом доверия }\end{array}$ \\
\hline $\mathrm{H} 2$ & $\begin{array}{l}\text { Механизм отзывов товара показывает более высокий уровень влияния на } \\
\text { доверие онлайн-потребителей с глобальным опытом онлайн-покупок через } \\
\text { восприятие качества информации, чем, на доверие онлайн-потребители, ко- } \\
\text { торые покупают только в своей стране }\end{array}$ \\
\hline $\mathrm{H} 3$ & $\begin{array}{l}\text { Механизм возврата (обмена) товаров, который предоставляет справедливый } \\
\text { процесс возврата (обмена) товара, обеспечивает интерактивную, процедур- } \\
\text { ную и дистрибутивную справедливость, показывает положительное отно- } \\
\text { шение с эффектом доверия }\end{array}$ \\
\hline $\mathrm{H} 4$ & $\begin{array}{l}\text { Механизм возврата (обмена) товаров, который позволяет онлайн- } \\
\text { потребителям воспринять контроль над возвратом (обменом) товаров, пока- } \\
\text { зывает положительное отношение с эффектом доверия }\end{array}$ \\
\hline H5 & $\begin{array}{l}\text { Механизма возврата (обмена) товара показывает более высокий уровень } \\
\text { влияние на доверие онлайн-потребителей с глобальным опытом онлайн- } \\
\text { покупок через восприятие справедливости, чем на доверие онлайн- } \\
\text { потребителей, которые покупают только в своей стране }\end{array}$ \\
\hline H6 & $\begin{array}{l}\text { Механизма возврата (обмена) товара показывает более высокий уровень } \\
\text { влияние на доверие онлайн-потребителей с глобальным опытом онлайн- } \\
\text { покупок через восприятие контроля, чем на доверие онлайн-потребителей, } \\
\text { которые покупают только в своей стране }\end{array}$ \\
\hline H7 & $\begin{array}{l}\text { Механизм обеспечения онлайн-гарантии, который предоставляет качест- } \\
\text { венный сетевой сервис онлайн-потребителям, включая информацию о пре- } \\
\text { дупреждении и надежные сигналы об обеспечении безопасности, показыва- } \\
\text { ет положительное отношение с эффектом доверия }\end{array}$ \\
\hline H8 & $\begin{array}{l}\text { Механизма обеспечения онлайн-гарантии показывает более высокий уро- } \\
\text { вень влияния на доверие онлайн-потребителей с глобальным опытом он- } \\
\text { лайн-потребителей через восприятие качества сетевого сервиса, чем на до- } \\
\text { верие онлайн-потребителей, которые покупают только в своей стране }\end{array}$ \\
\hline H9 & $\begin{array}{l}\text { Восприятие социального присутствия онлайн-потребителей, которое повы- } \\
\text { шается благодаря механизмам формирования доверия, показывает положи- } \\
\text { тельное отношение с эффектом доверия }\end{array}$ \\
\hline H10 & $\begin{array}{l}\text { Восприятие социального присутствия оказывает более высокий уровень } \\
\text { влияния на доверие онлайн-потребителей с глобальным опытом онлайн- } \\
\text { потребителей, чем на доверие онлайн-потребителей, которые покупают } \\
\text { только в своей стране }\end{array}$ \\
\hline
\end{tabular}

\section{Заключение}

Построенная модель основана на результатах проведенного нами исследования теоретических и эмпирических работ в области доверия онлайнпотребителей и самого феномена электронной розничной торговли $[24,33]$. В итоге мы установили, что широко известные модели формирования доверия онлайн-потребителей пока не совершенны, нами отмечен ряд противоречий и разрывов. Мы расширили модель D. Gefen и D.W. Straub, встроив в нее три механизма формирования доверия (механизм отзывов товаров, механизм возврата/обмена товара, механизм обеспечения онлайн-гарантии), рассматривая их как самые важные предшественники доверия онлайн-потребителей. Также мы 
применили другую теоретическую рамку (теория «социальные аксиом») для встраивания в модель фактора культуры и включили глобальность опыта онлайн-покупок, что, на наш взгляд, даст более точные объяснения того, как формируется доверие онлайн-потребителей в условиях развития трансграничной электронной торговли и использования новых технологий.

\section{Список источников / References}

1. Mcknight D.H., Chervany N.L. What trust means in e-commerce customer relationships: an interdisciplinary conceptual typology. International Journal of Electronic Commerce, 2001, no. 6 (2), pp. 35-59.

2. Mcknight D.H., Choudhury V., Kacmar C. The impact of initial consumer trust on intentions to transact with a web site: a trust-building model. Journal of Strategic Information Systems, 2002, no. 11, pp. 297-323.

3. Gefen D. E-commerce: the role of familiarity and trust. Omega, 2000, no. 28 (6), no. 725-737.

4. Gefen D. Reflections on the dimensions of trust and trustworthiness among online consumers. ACM SIGMIS Database, 2002, no. 33 (3), pp. 38-53.

5. Gefen D., Straub D.W. Consumer trust in B2C e-Commerce and the importance of social presence: experiments in e-Products and e-Services. Omega, 2004, no. 32, pp. 407-424.

6. Pavlou P.A., Gefen D. Building effective online marketplaces with institution-based trust. Information Systems Research, 2004, no. 15 (1), pp. 37-59.

7. Palvia P. The role of trust in e-commerce relational exchange: A unified model. Information \& Management, 2009, no. 46, pp. 213-220.

8. Jung Kun Park, Frances Gunn, Sang-Lin Han. Multidimensional trust building in eretailing: Cross-cultural differences in trust formation and implications for perceived risk. Journal of Retailing and Consumer Services, 2012, no. 19, pp. 304-312.

9. Hong I.B., Cha H.S. The mediating role of consumer trust in an online merchant in Predicting purchase intention. International Journal of Information Management, 2013, no. 33, pp. 927-939.

10. Kim S., Park H. Effects of various characteristics of social commerce (s-commerce) on consumers' trust and trust performance. International Journal of Information Management, 2013, no. 33, pp. 318-332.

11. Toufaily E., Souiden N., Ladhari R. Consumer trust toward retail websites: Comparison between pure click and click-and-brick retailers. Journal of Retailing and Consumer Services, 2013, no. 20, pp. 538-548.

12. Jarvenpaa S.L., Tractinsky N. Consumer trust in an internet store: a cross-cultural validation. Journal of Computer Mediated Communication, 1999, no. 5 (2), pp. 1-35.

13. Thompson S.H. Teo., Jing L. Consumer trust in e-commerce in the United States, Singapore and China. Omega, 2007, no. 35, pp. 22-38.

14. Danny, Tengti Kao. The Impact of Transaction Trust on Consumers' Intentions to Adopt M-Commerce: A Cross-Cultural Investigation. Cyber psychology and behavior, 2009, no. 12 (2), pp. 225-229.

15. Pitney Bowes Survey Finds Majority of Global Consumers Shop Cross-Border, 2016. Available at: https://www.businesswire.com/news/home/20161011006722/en/PitneyBowes-Survey-Finds-Majority-Global-Consumers

16. Bright Local. Local consumer review survey в 2016 г. Available at: http://www.brightlocal.com/learn/local-consumer-review-survey (accessed 16.05.2016).

17. Жалуются первые десять горячих точек национальной розничной электронной коммерции 2017 г. [2017年（上）全国零售电商十大热点被投诉问题, 100ec]. Available at: http://www.100ec.cn/zt/upload_data/17tsjc/17tsjc.pdf 
18. World Payments Report 2017, World pay. Available at: https://worldpay.globalpaymentsreport.com/wpcontent/uploads/reports/GPR-English2017.pdf

19. Consumers trust survey report, my pinpad 2017. Available at: https://mypinpad.com/wpontent/uploads/2017/07/MYPINPAD_ConsumerTrust_Report.p df

20. Количество зарубежных покупателей на платформе AliExpress в апреле 2017 года превысило 100 миллионов [2017年4月全球速卖通平台海外买家数破亿 中商情报网]. Available at: http://www.askci.com/news/hlw/20170416/14385796079.shtml

21. Техническая документация по международному трансграничному маркетингу 2018 г. [Forrester\&Facebook：2018全球跨境电商营销白皮书，互联网数据中心-199IT] Available at: http://www.199it.com/archives/746009.html

22. Chai L., Pavlou P. Customer relationship management.com: a cross-cultural empirical investigation of electronic commerce. AMCIS, 2002, no. 70, pp. 483-491.

23. Hofstede G. Culture's consequences: International differences in work-related values. Beverly Hills: Sage, 1980.

24. Тюрина Е.А., Цао Цзыин. Сравнительное исследование доверия потребителей в электронной розничной торговле в России и Китае. Материалы первой международной научно-практической конференции «Трансграничные рынки товаров и услуг: проблемы исследования». Владивосток, ДВФУ, 2015. [Tyurina E.A., Cao ZiYing. Sravnitel'noe issledovanie doveriya potrebitelej v elektronnoj roznichnoj torgovle v Rossii i Kitae [A Comparative Study of Consumer Confidence in Electronic Retail in Russia and China]. Materialy pervoj mezhdunarodnoj nauchno-prakticheskoj konferencii «Transgranichnye rynki tovarov i uslug: problemy issledovaniya» [Materials of the first international scientific-practical conference "Cross-border markets for goods and services: research problems."]. Vladivostok, FEFU, 2015.

25. Bonifield C., Cole C. Schultz R.L. Product returns on the Internet: A case of mixed signals? Journal of Business Research, 2010, no. 63, pp. 1058-1065.

26. Pei Z., Paswan A., Yan R. E-tailer's return policy, consumer's perception of return policy fairness and purchase intention. Journal of Retailing and Consumer Services, 2014, no. 21, pp. 249-257.

27. Рынок Интернет-торговли в России 2017, АКИТ. Available at: http://www.akit.ru/wpcontent/uploads/2018/04/-1.pdf

28. Отчет по исследованию трансграничной электронной торговли Китая за 2012-2013 гг. [2012-2013年中国跨境电商市场研究报告-百度文库] Available at: https://wenku.baidu.com/view/30b3053414791711cc7917c6.html

29. Исследование китайских трансграничных онлайн-потребителей 2014 г. [中国跨境网络购物用户研伊动力网] Available at: http://www.ebrun.com/20150430/132810_2.shtml

30. Отчет по исследованиям трансграничных онлайн-потребителей в Китае за 20152016 [2015-2016年中国跨境网购用户研究报告] Available at: http://report.iresearch.cn/report_pdf.aspx?id=2553

31. Fang L., Dixon M., Murphy J. Exploring online buying and online trust in China. Asia Pacific Advances in Consumer Research, 2002, no. 5, pp. 336-342.

32. Gounaris Spiros P., Venetis K. Trust in industrial service relationships: Behavioral consequences, antecedents and the moderating effect of the duration of the relationship. Journal of Services Marketing, 2002, no. 16 (7), pp. 636-655.

33. Цао Цзыин. Механизмы формирования доверия онлайн-потребителей в сфере электронной розничной торговли на примере Китая и России. Известия ДВФУ. Экономика и управление, 2018, №4, сc. 72-91. [Cao ZiYing. Mekhanizmy formirovaniya 
doveriya onlajn-potrebitelej $\mathrm{v}$ sfere elektronnoj roznichnoj torgovli na primere Kitaya i Rossii [Mechanisms for building online consumer confidence in electronic retail using the examples of China and Russia]. Izvestiya DVFU. Ekonomika i upravlenie $=$ The Bulletin of Far Eastern Federal University. Economics and Management, 2018, no. 4, pp. 72-91.]

34. Donath J. Signals in social supernets. Journal of Computer-Mediated Communication, 2007, no. 13 (1), pp. 231-251.

35. Metzger M.J. Effects of site, vendor, and consumer characteristics on web site trust and disclosure. Communication Research, 2006, no. 33 (3), pp. 155-179.

36. Spence M. Job market signaling. Quarterly Journal of Economics, 1973, no. 87 (3), pp. 355-374.

37. Cronin J.J., Taylor S.A. Measuring Service Quality: A Reexamination and Extension. Journal of Marketing, 1992, no. 56 (3), pp. 55-68.

38. Parasuraman A., Zeithaml V.A., Berry L.L. SERVQUAL: a multiple-item scale for measuring consumer perceptions of service quality. Journal of Retailing, 1988, no. 64, pp. 1240.

39. Rahman M.S., Khan A.H., Haque M.A Conceptual Study on the Relationship between Service Quality towards Customer Satisfaction: Servqual and Gronroos's Service Quality Model Perspective. Asian Social Science, 2012, no. 8 (13), pp. 201-210.

40. Adams J.S. Inequity in social exchange. Advances in experimental social psychology, 1965, no. 2, pp. 267-299.

41. Bies R.J., Moag J.S. Interactional justice: Communication criteria of fairness. Research on negotiation in organizations, 1986, no. 1, pp. 43-55.

42. Ajzen I. From intentions to actions: A theory of planned behavior. Action-control: From cognition to behavior, 1985, no. 2, pp. 11-39.

43. Kimery K.M., Cord M.Mc. Third party assurances: the road to trust in online retailing, Proceedings of the 35th Hawaii. International Conference on System Sciences, 2002, no. 4 (2), pp. 63-82.

44. Kim D.J. Sivasailam N., Rao H.R. Information assurance in B2C Websites for information goods/services. Electronic Markets, 2004, no. 14 (4), pp. 344- 359.

45. Miyazaki A.D., Fernandez A. Consumer perception of privacy and security risks for online shopping. The Journal of Consumer Affairs, 2001, no. 35 (1), pp. $27-44$.

46. Noteberg A., Christiaanse E., Wallage P. Consumer trust in electronic channels: the impact of electronic commerce assurance on consumers' purchasing likelihood and risk perceptions. E-Service Journal, 2003, no. 2 (2), pp. 46- 67.

47. Отчет о отношении онлайн-потребителей к конфиденциальности и безопасности 2017 г. [2017年消费者对隐私和安全态度报告-互联网数据中心-199IT] Available at: http://www.199it.com/archives/591073.html

48. Ladhari R. Developing e-service quality scales: A literature review. Journal of Retailing and Consumer Services, 2010, no. 17, pp. 464-477.

49. Parasuraman A., Zeithaml V.A., Malhotra A. E-S-Qual: a multiple-item scale for assessing electronic service quality. Journal of Service Research, 2005, no. 7 (3), pp. $213-$ 233.

50. Thaichon P., Lobo A., Prentice C. Thu Nguyen Quach, the development of service quality dimensions for internet service providers: Retaining customers of different usage patterns. Journal of Retailing and Consumer Services, 2014, no. 21, pp. 1047-1058.

51. Лебедева Н. М., Татарко А.Н. Культура как фактор общественного прогресса. Москва, 3АО «Юстицинформ», 2009. 408 с. [Lebedeva N. M., Tatarko A.N. Kul'tura kak faktor obshchestvennogo progressa [Culture as a factor in social progress]. Moscow, Justicinform Publ., 2009. 408 p.]

52. Leung K., Bond M.H., Reimel de Carrasquel S. Muñoz C. Hernández M., Murakami F., Yamaguchi S., Bierbrauer G., Singelis T.M. Social axioms: The search for universal dimensions of general beliefs about how the world functions. Journal of Cross-Cultural Psychology, 2002, no. 33, pp. 286-302. 
53. Kang J. Building Trust between Consumers and Corporations: The Role of Consumer Perceptions of Transparency and Social Responsibility. Journal of Business Ethics, 2014, no. 125 , pp. 253-265.

54. Jin B., Park J.Y. The Moderating Effect of Online Purchase Experience on the Evaluation of Online. Advances in Consumer Research, 2006, no. 33, pp. 203-211.

55. Kim H.J. Ahn Soo-K. Forney J.A., Shifting paradigms for fashion: from total to global to smart consumer experience. Fashion and Textiles, 2014, no. 1 (15), pp. 2-16.

56. Global Connected Commerce - Nielsen 2016 г. Available at: https://www.nielsen.com/wp-content/uploads/sites/3/2019/04/connected-commercereport-jan-2016.pdf

57. Интернет-торговля в России 2018, DataInsight. Available at: http://datainsight.ru/ecommerce_2018

\section{Сведения об авторах / About authors}

Цао Цзыин, ассистент Департамента экономических наук, исследователь Лаборатории исследования поведения потребителей на рынках стран АТР, Школа экономики и менеджмента, Дальневосточный федеральный университет. 690922 Россия, г. Владивосток, о-в Русский, кампус ДВФУ, корпус G, каб. G320.E-mail: tcao.tc@dvfu.ru

Cao ZiYing, Assistant, the Department of Economic Sciences, Researcher, the Laboratory for Consumer Behavior Research in the Asia-Pacific Markets, School of Economics and Management, Far Eastern Federal University. Office G320, Building G, FEFU campus, Russky Island, Vladivostok, Russia 690922.E-mail:tcao.tc@dvfu.ru

Тюрина Елена Александровна, кандидат экономических наук, доцент, доцент кафедры менеджмента, исследователь междисциплинарной лаборатории «Far Eastern Climate Smart Lab», Школа экономики и менеджмента, Дальневосточный федеральный университет. 690922 Россия, г. Владивосток, о-в Русский, кампус ДВФУ, корпус G, каб. G612. ORCID ID: 0000-0002-62160697.E-mail: tyurina.ea@dvfu.ru

Elena A. Tyurina, Candidate of Economic Sciences, Associate Professor, the Department of Management, Researcher, the Interdisciplinary Laboratory "Far Eastern Climate Smart Lab", School of Economics and Management, Far Eastern Federal University. Office 612, Building G, FEFU campus, Russky Island, Vladivostok, Russia 690922. ORCID ID: 0000-0002-6216-0697.

E-mail: tyurina.ea@dvfu.ru

Филатов Александр Юрьевич, кандидат физико-математических наук, доцент, доцент Департамента экономических наук, научный руководитель Научно-исследовательской лаборатории моделирования социально-экономических процессов, Школа экономики и менеджмента, Дальневосточный федеральный университет. 690922 Россия, г. Владивосток, о-в Русский, кампус ДВФУ, корпус G, каб. G335. E-mail: filatov.aiu@dvfu.ru

Aleksander Yu. Filatov, Candidate of Physical and Mathematical Sciences, Associate Professor, the Department of Economic Sciences, Scientific Director, the Research Laboratory of Modeling SocioEconomic Processes, School of Economics and Management, Far Eastern Federal University. Office G335, Building G, FEFU campus, Russky Island, Vladivostok, Russia 690922.

E-mail: filatov.aiu@dvfu.ru 